\title{
Dynamic Pricing for Airline Revenue Management under Passenger Mental Accounting
}

\author{
Yusheng Hu, Jinlin Li, and Lun Ran \\ School of Management and Economics, Beijing Institute of Technology, Beijing 100081, China \\ Correspondence should be addressed to Yusheng Hu; huyusheng1981@163.com
}

Received 10 August 2014; Accepted 2 December 2014

Academic Editor: Fazal M. Mahomed

Copyright (C) 2015 Yusheng Hu et al. This is an open access article distributed under the Creative Commons Attribution License, which permits unrestricted use, distribution, and reproduction in any medium, provided the original work is properly cited.

\begin{abstract}
Mental accounting is a far-reaching concept, which is often used to explain various kinds of irrational behaviors in human decision making process. This paper investigates dynamic pricing problems for single-flight and multiple flights settings, respectively, where passengers may be affected by mental accounting. We analyze dynamic pricing problems by means of the dynamic programming method and obtain the optimal pricing strategies. Further, we analytically show that the passenger mental accounting depth has a positive effect on the flight's expected revenue for the single flight and numerically illustrate that the passenger mental accounting depth has a positive effect on the optimal prices for the multiple flights.
\end{abstract}

\section{Introduction}

In airline revenue management, revenue maximization could be attained by dynamic pricing and capacity control [1-6], in which dynamic pricing is regarded as the engine and core technique of airline revenue management and plays a very important role in improving airline's revenue. Obviously, the rapid development of Internet has enabled airlines to collect enormous pieces of market information. Therefore, airlines could timely change flight prices to increase their revenue according to the market demand and the remaining seat level of flight. The success of dynamic pricing depends on whether the passengers' response to price change can be anticipated properly.

Most classical dynamic pricing models suppose that passenger behavior is rational [7]; that is, a passenger chooses the flight as soon as the flight price is below his/her valuation. However, in the practical condition, passengers usually exhibit some irrationality; for example, passenger could mentally aggregate or segregate purchase systematically in view of factors such as time or some uncertain events before making valuations. Thus, it is important to examine passengers' decision processes and then set the corresponding flight price for the airline. Mental accounting first studied by Thaler has a far-reaching impact on finance and behavior economics [8], which is often used to interpret all kinds of irrational behaviors in the decision making process. It refers to the tendency that people divide their assets into several independent accounts based on various subjective criteria, such as the source of the assets and the intention of every account. In the light of mental accounting, different levels of the utility are allocated to every account by individuals, which influences their spending decisions.

In recent years, as the customer behavior issue has been introduced into the operations management field, modeling customer behavior increasingly attracts extensive attention in the area of dynamic pricing and revenue management [9-12].

In the customer choice behavior model, dynamic pricing of multiple products is studied extensively. Zhang and Cooper [13] develop a Markov decision process formulation of a dynamic pricing problem with multiple parallel flights, taking into account customer choice among the flights. Akçay et al. [14] use stochastic dynamic programming method to approach the joint dynamic pricing issue of multiple vertically differentiated products and characterize the optimal prices. Lin and Sibdari [15] establish a game model to describe the dynamic pricing competition between companies that sell substitutable products and prove the existence of the Nash equilibrium prices. Suh and Aydin [16] investigate the dynamic pricing problem of two substitutable products based 
on multinomial logit (MNL) choice model, and the results indicate that, under the optimal pricing strategy, the marginal revenue function increases with the remaining time and decreases with the inventory of each product. Dong et al. [17] apply MNL choice model to study dynamic pricing and inventory control of substitutable products.

In the strategic customer model, dynamic pricing problems are still under development. Aviv and Pazgal [18] focus on the dynamic pricing problem of seasonal goods in the presence of strategic customer. Levin et al. [19, 20] study the influence of strategic customer behavior on the dynamic pricing policies in the case of monopoly and oligopoly, respectively. Levina et al. [21] consider dynamic pricing based on online learning and strategic customers. Chen and Zhang [22] solve the dynamic targeted pricing problem with strategic customers and show that the dynamic targeted pricing can get more profits for competing firms, if they actively pursue customer recognition on the basis of customer purchase records.

The concept of customer mental accounting has been widely studied in the operations management literature. Liao and Chu [23] utilize the mental accounting theory to study how customers' economic psychology associated with purchasing a new product is influenced when a consciousness of the possibility of online retail is aroused. The result indicates that customers' consciousness of the retail value of already possessed product can affect their decision to buy a new product. Erat and Bhaskaran [24] formulate a simple model to investigate how mental accounting associated with a base product influences a customer's add-on purchase decision. Chen et al. [25] study the inventory order quantity problem based on the principle of mental accounting. In the existing literatures related to mental accounting, researchers have paid less attention to dynamic pricing problem.

In this paper, we investigate the multiperiod dynamic pricing problems for a single flight and multiple flights in the case of a monopoly airline selling the ticket to passengers who could be affected by mental accounting. We explicitly model the passenger dynamic choice process by MNL choice model, in which the probability of purchasing a ticket is specified as a function of mental accounting depth. At each decision period, airline dynamically determines the flight price in order to maximize the expected revenue over the booking horizon. Given the passenger choice model, we formulate the dynamic pricing problems using the dynamic programming method for a single flight and multiple flights in the presence of passenger mental accounting. Then we obtain the optimal pricing strategies. The optimal prices are significantly affected by the depth of mental accounting. Furthermore, we numerically demonstrate the positive effect of passenger mental accounting on airline's dynamic pricing strategies.

The model of passenger mental accounting in this paper complements the related research on behavioral operations, and the aim of this paper is to establish a manageable modeling framework that embodies mental accounting. The remainder of this paper is organized as follows. In Section 2, we present a single-flight model formulation and characterize the optimal price. In Section 3, we make further discussion on the dynamic pricing problem of multiple flights followed by a numerical study presented in Section 4 . Finally, we summarize this paper in Section 5.

\section{Dynamic Pricing for a Single Flight}

In this section, we investigate a single-flight dynamic pricing problem. The model can be regarded as a building block of the multiple-flight case. Consider a single-leg flight with capacity $C$. We divide the booking horizon into $T$ discrete time periods such that there is at most one passenger arrival in each time period and suppose that each passenger books no more than one ticket. The time period is counted in reverse chronological order. Therefore, the first time period is $T$ and the last time period is 1 . In each time period, passengers arrive independently, and there is one passenger arrival with probability $\lambda$. The airline's goal is to maximize the expected revenue from the booking horizon subject to the capacity constraint.

2.1. Dynamic Pricing Formulation. We first present a singleflight decision framework to capture passenger's mental accounting. Consider a potential passenger deciding whether to purchase a flight ticket or not to buy at all. Suppose that payment of purchasing the flight ticket is classified in mental accounting, such as travel mental accounting. Let $\gamma(\geq 0)$ be the trigger increment of mental accounting. When $\gamma>0$, a passenger possesses this mental accounting; otherwise, a passenger does not possess this mental accounting. We could interpret $\gamma$ as the depth of mental accounting, because it captures how strongly each passenger is influenced by mental accounting.

Assume that the utility from passenger's purchasing is $U$ and the utility from nonpurchasing is $U_{0}$. In detail, $U$ depends on the ticket price and passenger's valuation for the ticket, and $U_{0}$ depends on the passenger's external option such as the future purchase and the change of travel plans. Given $U$ and $U_{0}$, the perfectly rational passenger will purchase the flight ticket if and only if $U \geq U_{0}$ [2]. However, when mental accounting is considered, the passenger will purchase the flight ticket if and only if

$$
U+\gamma \geq U_{0}
$$

We describe the passenger choice process by MNL choice model. The MNL choice model has a wide range of applications in the marketing literature, since it is analytically tractable, considerably accurate, and could be estimated easily by standard statistical methods [26]. The distinction of application is that passenger mental accounting is captured in our model. At the beginning of each period, airline sets its flight price, and then a passenger decides whether to purchase one or not to purchase at all. When facing a price $p_{t}$, for each passenger, the utility from purchasing a flight ticket is equal to

$$
U_{t}=a-p_{t}+\gamma+\zeta
$$

where $a$ is the quality index of the flight, which describes the service level, brand image, and the popularity of the flight, 
and $\zeta$ is a Gumbel random variable with shift parameter zero and scale parameter one. The utility from nonpurchasing is $U_{0}$; without loss of generality, we suppose that $U_{0}$ is normalized to zero.

Let $q_{t}$ be the probability that a passenger chooses to purchase the flight ticket at price $p_{t}$ in each period $t$. Based on MNL choice model, we can write the purchase probability $q_{t}$ as

$$
q_{t}=\frac{e^{a-p_{t}+\gamma}}{1+e^{a-p_{t}+\gamma}}
$$

therefore, in each period $t$, the probability that a passenger decides not to purchase any flight ticket is $q_{0 t}=1-q_{t}$.

Given the remaining seat level $x_{t}$, let $V_{t}\left(x_{t}\right)$ be the optimal expected revenue from period $t$ to the last period. Then we formulate the single-flight dynamic pricing problem as in the following Bellman equation:

$$
\begin{aligned}
& V_{t}\left(x_{t}\right)=\max _{p \in R^{+}}\{ \lambda\left[q_{t}\left(p_{t}+V_{t-1}\left(x_{t}-1\right)\right)+q_{0 t} V_{t-1}\left(x_{t}\right)\right] \\
&\left.+(1-\lambda) V_{t-1}\left(x_{t}\right)\right\} \\
&=\max _{p \in R^{+}}\left\{\lambda\left[q_{t}\left(p_{t}+V_{t-1}\left(x_{t}-1\right)-V_{t-1}\left(x_{t}\right)\right)\right]\right. \\
&\left.+V_{t-1}\left(x_{t}\right)\right\}
\end{aligned}
$$

with boundary conditions $V_{0}\left(x_{t}\right)=0$, for all $x_{t}$, and $V_{t}(0)=0$, for $t=1,2, \ldots, T$.

Let $\Delta V_{t-1}(x)=V_{t-1}(x)-V_{t-1}(x-1)$ represent the marginal expected revenue with remaining seat level $x$ in period $t-1$. Using this notation, we can rewrite (4) as follows:

$$
V_{t}\left(x_{t}\right)=\max _{p \in R^{+}}\left\{\lambda q_{t}\left[p_{t}-\Delta V_{t-1}\left(x_{t}\right)\right]+V_{t-1}\left(x_{t}\right)\right\} .
$$

2.2. Optimal Pricing Strategy. In the following section, we describe the optimal pricing strategy. Before obtaining the optimal price and expected revenue, we first rewrite $p_{t}$ as a function of the purchase probability $q_{t}$. From (3), we have

$$
p_{t}=a+\gamma-\ln \left(q_{t}\right)+\ln \left(1-q_{t}\right) \text {. }
$$

Then we can reformulate the expected revenue function as

$$
\begin{aligned}
V_{t}\left(x_{t}\right)=\max _{p \in R^{+}}\{ & \lambda q_{t}\left[a+\gamma-\ln \left(q_{t}\right)+\ln \left(1-q_{t}\right)-\Delta V_{t-1}\left(x_{t}\right)\right] \\
& \left.+V_{t-1}\left(x_{t}\right)\right\} .
\end{aligned}
$$

Next, we define function

$$
\begin{aligned}
\varphi\left(x_{t}, p_{t}\right) & =\lambda q_{t}\left[p_{t}-\Delta V_{t-1}\left(x_{t}\right)\right] \\
& =\lambda q_{t}\left[a+\gamma-\ln \left(q_{t}\right)+\ln \left(1-q_{t}\right)-\Delta V_{t-1}\left(x_{t}\right)\right] .
\end{aligned}
$$

In Theorem 1 , we can show that $\varphi\left(x_{t}, p_{t}\right)$ is a concave function of $q_{t}$ in the case of a single flight.

Theorem 1. Function $\varphi\left(x_{t}, p_{t}\right)$ is concave in $q_{t}$.
Proof. Because $\Delta V_{t-1}\left(x_{t}\right)$ is not influenced by $q_{t}$, we view it as a constant. Taking the first derivative of $\varphi\left(x_{t}, p_{t}\right)$ with respect to $q_{t}$ yields

$$
\begin{aligned}
& \frac{\partial \varphi\left(x_{t}, p_{t}\right)}{\partial q_{t}} \\
& =\lambda\left[a+\gamma-\ln \left(q_{t}\right)+\ln \left(1-q_{t}\right)-\Delta V_{t-1}\left(x_{t}\right)-\frac{1}{1-q_{t}}\right] .
\end{aligned}
$$

In order to derive the concavity of $\varphi\left(x_{t}, p_{t}\right)$, we take the second derivative of $\varphi\left(x_{t}, p_{t}\right)$ with respect to $q_{t}$, which leads to

$$
\frac{\partial^{2} \varphi\left(x_{t}, p_{t}\right)}{\partial q_{t}^{2}}=-\lambda \frac{1}{q_{t}\left(1-q_{t}\right)^{2}}<0
$$

It then follows that $\varphi\left(x_{t}, p_{t}\right)$ is concave in $q_{t}$.

From Theorem 1, we can also conclude that the expected revenue function $V_{t}\left(x_{t}\right)$ is concave in $q_{t}$. In Theorem 2 , we can derive the optimal price $p_{t}^{*}\left(x_{t}\right)$ to problem (5).

Theorem 2. For a given remaining seat level $x_{t}$ in period $t$, the optimal price is set to

$$
p_{t}^{*}\left(x_{t}\right)=\Delta V_{t-1}\left(x_{t}\right)+\omega\left(t, x_{t}\right),
$$

where $\omega\left(t, x_{t}\right)$ is the unique solution such that

$$
\left[\omega\left(t, x_{t}\right)-1\right] e^{\omega\left(t, x_{t}\right)}-e^{a+\gamma-\Delta V_{t-1}\left(x_{t}\right)}=0 .
$$

Furthermore, the optimal expected revenue is given by

$$
V_{t}^{*}\left(x_{t}\right)=\lambda \sum_{h=1}^{t}\left[\omega\left(h, x_{t}\right)-1\right] \text {. }
$$

Proof. From Theorem 1, we have known the concavity of $\varphi\left(x_{t}, p_{t}\right)$. In order to obtain the optimal price $p_{t}^{*}\left(x_{t}\right)$, we compute the first derivative of $\varphi\left(x_{t}, p_{t}\right)$ with respect to $q_{t}$. The first-order condition yields

$$
\begin{aligned}
& \frac{\partial \varphi\left(x_{t}, p_{t}\right)}{\partial q_{t}} \\
& =\lambda\left[a+\gamma-\ln \left(q_{t}\right)+\ln \left(1-q_{t}\right)-\Delta V_{t-1}\left(x_{t}\right)-\frac{1}{1-q_{t}}\right]=0 .
\end{aligned}
$$

Defining $\omega\left(t, x_{t}\right)=1 /\left(1-q_{t}\right)$ and then substituting $\omega\left(t, x_{t}\right)$ into (14), we have

$$
q_{t}=\frac{1}{\omega\left(t, x_{t}\right)} e^{a+\gamma-\Delta V_{t-1}\left(x_{t}\right)-\omega\left(t, x_{t}\right)} .
$$

Since $q_{t}=1-1 / \omega\left(t, x_{t}\right)$, we can get

$$
1-\frac{1}{\omega\left(t, x_{t}\right)}=\frac{1}{\omega\left(t, x_{t}\right)} e^{a+\gamma-\Delta V_{t-1}\left(x_{t}\right)-\omega\left(t, x_{t}\right)} .
$$


This means that $\omega\left(t, x_{t}\right)$ is one solution to

$$
\left[\omega\left(t, x_{t}\right)-1\right] e^{\omega\left(t, x_{t}\right)}-e^{a+\gamma-\Delta V_{t-1}\left(x_{t}\right)}=0 .
$$

We then show that $\omega\left(t, x_{t}\right)$ is the unique solution to (17).

Let $\Phi(\xi)=(\xi-1) e^{\xi}-e^{a+\gamma-\Delta V_{t-1}\left(x_{t}\right)}$; we immediately get $\Phi(1)<0, \Phi(+\infty)=+\infty$. Since $\partial \Phi(\xi) / \partial \xi=\xi e^{\xi}>0$ for any $\xi \in(1,+\infty)$, it follows that $\Phi(\xi)$ is monotonically increasing in $\xi$ for $\xi \in(1,+\infty)$. This indicates that $\omega\left(t, x_{t}\right) \in(1,+\infty)$ is the unique solution to (17). We then obtain the optimal solution $p_{t}^{*}\left(x_{t}\right)$ from $\omega\left(t, x_{t}\right)$. Substituting $q_{t}=1-1 / \omega\left(t, x_{t}\right)$ into (6), we can derive $p_{t}^{*}\left(x_{t}\right)=a+\gamma-\ln \left[\omega\left(t, x_{t}\right)-1\right]$. Rewriting the above expression, we have $\omega\left(t, x_{t}\right)-1=$ $e^{a+\gamma-p_{t}^{*}\left(x_{t}\right)}$. From (17), we then get

$$
p_{t}^{*}\left(x_{t}\right)=\Delta V_{t-1}\left(x_{t}\right)+\omega\left(t, x_{t}\right) \text {. }
$$

Substituting (18) into (5), we have

$$
V_{t}^{*}\left(x_{t}\right)=\lambda\left[\omega\left(t, x_{t}\right)-1\right]+V_{t-1}\left(x_{t}\right)
$$

Applying the boundary conditions, we can get

$$
V_{t}^{*}\left(x_{t}\right)=\lambda \sum_{h=1}^{t}\left[\omega\left(h, x_{t}\right)-1\right] \text {. }
$$

Theorem 2 describes the evolution of optimal prices. For convenience of exposition, $\omega\left(t, x_{t}\right)$ is defined as the revenue margin of selling one seat in period $t$; namely, $\omega\left(t, x_{t}\right)$ denotes the immediate contribution from selling one seat, and $\Delta V_{t-1}\left(x_{t}\right)$ denotes the future value of one remaining seat to the overall revenue. From (18), we can observe that the optimal price $p_{t}^{*}\left(x_{t}\right)$ depends on the interaction between the immediate contribution and the future value of one remaining seat. From (17), it is clear that an increase of $\omega\left(t, x_{t}\right)$ leads to a decrease of $\Delta V_{t-1}\left(x_{t}\right)$, which leads to complicated optimal price patterns.

Theorem 3 provides the effect of mental accounting depth on flight's expected revenue, considering no seat scarcity.

Theorem 3. Let $x_{t}$ denote the remaining seat level in period $t$; if $x_{t} \geq t$, then $V_{t}\left(x_{t}\right)$ is nondecreasing with respect to mental accounting depth $\gamma$.

Proof. To prove this theorem, we need to show that, for all $i \leq t, \Delta V_{i}\left(x_{t}\right)=0$ given that $x_{t}>t$.

First, let us consider $t=1$. Since $\Delta V_{0}(x)=0$ for all $x$, $\omega\left(1, x_{1}\right)$ is determined by solving

$$
\left[\omega\left(1, x_{1}\right)-1\right] e^{\omega\left(1, x_{1}\right)}-e^{a+\gamma}=0 .
$$

This implies that $\omega\left(1, x_{1}\right)$ is independent of $x_{1}$. So all $V_{1}\left(x_{1}\right)$ are equal for any $x_{1}>0$. It follows that $\Delta V_{1}\left(x_{1}\right)=V_{1}\left(x_{1}\right)-$ $V_{1}\left(x_{1}-1\right)=0$, if $x_{1}>1 ; \Delta V_{1}(1)=V_{1}(1)-0=V_{1}(1)$, if $x_{1}=1$.

Next, we consider $t=2$. Note that since $\Delta V_{1}\left(x_{2}\right)=0$ for $x_{2} \geq 2, \omega\left(2, x_{2}\right)$ for $x_{2} \geq 2$ is determined by solving

$$
\left[\omega\left(2, x_{2}\right)-1\right] e^{\omega\left(2, x_{2}\right)}-e^{a+\gamma}=0 .
$$

This means that all $\omega\left(2, x_{2}\right)$ are equal for any $x_{2} \geq 2$. Therefore, if $x_{2} \geq 2$, all $V_{2}\left(x_{2}\right)$ values are the same and $\Delta V_{2}\left(x_{2}\right)=V_{2}\left(x_{2}\right)-V_{2}\left(x_{2}-1\right)=0$ for any $x_{2}>2$. But if $x_{2}=2, \Delta V_{2}(2)=V_{2}(2)-V_{2}(1) \neq 0$.

Similarly, we can deduce that all $V_{t}\left(x_{t}\right)$ are equal for any $x_{t} \geq t$, and $\Delta V_{t}\left(x_{t}\right)=0$, if $x_{t}>t$. It follows that for all $i \leq$ $t \Delta V_{i}\left(x_{t}\right)=0$ given that $x_{t}>t$. From the above derivation process, we can conclude that all $\omega\left(i, x_{t}\right)$ are equal for all $i \leq t$, if $x_{t} \geq t$. We then prove the theorem.

Consider $t=1$. Since $V_{1}\left(x_{1}\right)$ is independent of $\gamma, V_{1}\left(x_{1}\right)$ is nondecreasing with respect to $\gamma$.

Consider $t \geq 2$ and $x_{t}>t$. The corresponding $\omega\left(t, x_{t}\right)$ is determined by the equation

$$
\left[\omega\left(t, x_{t}\right)-1\right] e^{\omega\left(t, x_{t}\right)}-e^{a+\gamma-\Delta V_{t-1}\left(x_{t}\right)}=0 .
$$

Let $g_{1}=\left[\omega\left(t, x_{t}\right)-1\right] e^{\omega\left(t, x_{t}\right)}, g_{2}=e^{a_{t}+\gamma-\Delta V_{t-1}\left(x_{t}\right)}$. Obviously, $g_{1}$ is monotonically increasing with respect to $\omega\left(t, x_{t}\right)$, and $g_{2}$ is monotonically increasing with respect to $\gamma$. Therefore, $\omega\left(t, x_{t}\right)$ is monotonically nondecreasing with respect to $\gamma$. Because all $\omega\left(i, x_{t}\right)$ are equal for any $i \leq t$, if $x_{t} \geq t$, we can easily verify that $V_{t}\left(x_{t}\right)$ is monotonically nondecreasing with respect to $\gamma$ from $(20)$.

\section{Dynamic Pricing for Multiple Flights}

In this section, we deal with a multiple-flight dynamic pricing problem. The airline's goal is to maximize the expected revenue from the booking horizon by setting the appropriate price for each flight in each period; in other words, in every period $t$, for each current remaining seat level $x_{t}=$ $\left(x_{1 t}, x_{2 t}, \ldots, x_{m t}\right)$, the airline must determine the price vector $p_{t}=\left(p_{1 t}, p_{2 t}, \ldots, p_{m t}\right)$.

3.1. Dynamic Programming Formulation. There are $m$ substitutable flights in a single origin-destination pair with capacity $c_{i}$ of flight $i$. The payments of different flights are classified among different mental accounts. The booking horizon is discrete and is divided into $T$ time periods such that there is at most one passenger arrival in each time period, and suppose that each passenger books no more than one ticket, and the time period runs backwards. Passengers arrive independently across time periods. Let $\lambda$ be the probability of a passenger arrival in each period. The utility that passenger would obtain from the purchase of flight $j$ is $U_{j}(j=1,2, \ldots, m)$ and the utility from nonpurchasing is $U_{0}$; without loss of generality, we suppose that $U_{0}$ is normalized to zero.

Passengers choose among the substitutable flights or choose nothing; the probability that a passenger chooses flight $j$ is $q_{j}\left(p_{t}\right) ; q_{0}\left(p_{t}\right)$ represents the probability that an arriving passenger does not make any purchase. In period $t$, we define passenger's utility from the purchase of flight $j$ at price $p_{j t}$ as

$$
U_{j t}=a_{j}-p_{j t}+b_{j} \gamma_{j}+\zeta_{j}
$$

where $a_{j}$ represents the quality level of flight $j, b_{j}$ is the mental accounting coefficient, which represents the satisfaction degree that a passenger derives from choosing flight $j$ and 
$\sum_{j=1}^{m} b_{j}=1$, and $\zeta_{j}$ is a Gumbel random variable with shift parameter zero and scale parameter one. Defining $b_{j} \gamma_{j}=\Lambda_{j}$, based on MNL choice model, we conclude that a passenger will choose flight $j$ with probability

$$
q_{j t}=\frac{e^{a_{j}-p_{j t}+\Lambda_{j}}}{1+\sum_{k=1}^{m} e^{a_{k}-p_{k t}+\Lambda_{k}}}
$$

and the passenger will decide to purchase nothing with probability $q_{0 t}=1 /\left(1+\sum_{k=1}^{m} e^{a_{k}-p_{k t}+\Lambda_{k}}\right)$.

Let $V_{t}\left(x_{t}\right)$ be the optimal expected revenue from period $t$ to the last period. Then we can use the following dynamic programming to formulate the multiple-flight dynamic pricing problem:

$$
\begin{aligned}
& V_{t}\left(x_{t}\right) \\
& =\max _{p_{t} \in R_{m}^{+}}\left\{\lambda\left[\sum_{j=1}^{m} q_{j t}\left(p_{j t}+V_{t-1}\left(x_{t}-\varepsilon_{j}\right)\right)+q_{0 t} V_{t-1}\left(x_{t}\right)\right]\right. \\
& \left.+\left(1-\lambda_{t}\right) V_{t-1}\left(x_{t}\right)\right\} \\
& =\max _{p_{t} \in R_{m}^{+}}\left\{\lambda\left[\sum_{j=1}^{m} q_{j t}\left(p_{j t}+V_{t-1}\left(x_{t}-\varepsilon_{j}\right)-V_{t-1}\left(x_{t}\right)\right)\right]\right. \\
& \left.+V_{t-1}\left(x_{t}\right)\right\},
\end{aligned}
$$

with boundary conditions $V_{0}\left(x_{t}\right)=0, V_{t}(0)=0$ for $t=1,2, \ldots, T$, where $\varepsilon_{j}$ denotes a $n$-vector with the $j$ th component 1 and zeros elsewhere.

For the expected revenue function $V_{t}\left(x_{t}\right)$, define the marginal revenue of flight $j$ by

$$
\begin{gathered}
\Delta_{x_{j}} V_{t-1}\left(x_{t}\right)=V_{t-1}\left(x_{t}\right)-V_{t-1}\left(x_{t}-\varepsilon_{j}\right) \\
\text { for } t=1,2, \ldots, T, \quad j=1,2, \ldots, m .
\end{gathered}
$$

Using this notation, we can rewrite (26) as follows:

$$
\begin{gathered}
V_{t}\left(x_{t}\right)=\max _{p_{t} \in R_{m}^{+}}\left\{\lambda\left[\sum_{j=1}^{m} q_{j t}\left(p_{j t}-\Delta_{x_{j}} V_{t-1}\left(x_{t}\right)\right)\right]\right. \\
\left.+V_{t-1}\left(x_{t}\right)\right\} .
\end{gathered}
$$

3.2. Optimal Pricing Strategy. We next investigate the optimal pricing strategy. Before obtaining the optimal price, we first rewrite the price of each flight as a function of passenger's choice probability. From $q_{0 t} / q_{j t}=1 / e^{a_{j}-p_{j t}+\Lambda_{j}}$, we have $p_{j t}=$ $a_{j}+\Lambda_{j}-\ln \left(q_{j t} / q_{0 t}\right)$; substituting $p_{j t}$ into (28), we can derive

$$
\begin{aligned}
& V_{t}\left(x_{t}\right) \\
& =\max _{p_{t} \in R_{m}^{+}}\left\{\lambda\left[\sum_{j=1}^{m} q_{j t}\left(a_{j}+\Lambda_{j}-\ln \frac{q_{j t}}{q_{0 t}}-\Delta_{x_{j}} V_{t-1}\left(x_{t}\right)\right)\right]\right. \\
& \left.+V_{t-1}\left(x_{t}\right)\right\} .
\end{aligned}
$$

To facilitate description of structural properties, we define

$$
\begin{aligned}
\psi\left(x_{t}, p_{t}\right) & =\lambda\left[\sum_{j=1}^{m} q_{j t}\left(p_{j t}-\Delta_{x_{j}} V_{t-1}\left(x_{t}\right)\right)\right] \\
& =\lambda\left[\sum_{j=1}^{m} q_{j t}\left(a_{j}+\Lambda_{j}-\ln \frac{q_{j t}}{q_{0 t}}-\Delta_{x_{j}} V_{t-1}\left(x_{t}\right)\right)\right],
\end{aligned}
$$

which can be proved to be concave in $q_{t}=\left(q_{0 t}, q_{1 t}, q_{2 t}, \ldots\right.$, $\left.q_{m t}\right)$ in Theorem 4. Intuitively, $\psi\left(x_{t}, p_{t}\right)$ is the expected additional revenue realized in period $t$ by booking a single unit of remaining seat level $x_{t}$ at price $p_{t}$. We can interpret $\psi\left(x_{t}, p_{t}\right)$ as the marginal revenue of time at the remaining seat level $x_{t}$ in period $t$ when flight prices are set at $p_{t}$. Consequently, we set the optimal prices in order to maximize $\psi\left(x_{t}, p_{t}\right)$. Therefore, exploring the structural properties of $\psi\left(x_{t}, p_{t}\right)$ is the key to find the optimal prices. We discuss the structural properties of $\psi\left(x_{t}, p_{t}\right)$ as follows.

Theorem 4. Function $\psi\left(x_{t}, p_{t}\right)$ is concave in $q_{t}$.

Proof. Taking the second derivatives of $\psi\left(x_{t}, p_{t}\right)$, we can derive

$$
\begin{gathered}
\frac{\partial^{2} \psi\left(x_{t}, p_{t}\right)}{\partial q_{0 t}^{2}}=-\lambda \sum_{j=1}^{m} \frac{q_{j t}}{\left(q_{0 t}\right)^{2}}, \\
\frac{\partial^{2} \psi\left(x_{t}, p_{t}\right)}{\partial q_{0 t} \partial q_{k t}}=\frac{\partial^{2} \psi\left(x_{t}\right)}{\partial q_{k t} \partial q_{0 t}}=\frac{\lambda}{q_{0 t}}, \\
\text { for } k=1,2, \ldots, m . \\
\frac{\partial^{2} \psi\left(x_{t}, p_{t}\right)}{\partial q_{k t}^{2}}=-\frac{\lambda}{q_{k t}}, \quad \text { for } k=1,2, \ldots, m, \\
\frac{\partial^{2} \psi\left(x_{t}, p_{t}\right)}{\partial q_{l t} \partial q_{k t}}=\frac{\partial^{2} \psi\left(x_{t}, p_{t}\right)}{\partial q_{k t} \partial q_{l t}}=0, \quad \text { for } l \neq k .
\end{gathered}
$$


Then the Hessian matrix of $\psi\left(x_{t}, p_{t}\right)$ follows as

$$
\begin{aligned}
& H\left(\psi\left(x_{t}, p_{t}\right)\right) \\
& =\left(\begin{array}{ccccc}
-\lambda \sum_{j=1}^{m} \frac{q_{j t}}{\left(q_{0 t}\right)^{2}} & \frac{\lambda}{q_{0 t}} & \frac{\lambda}{q_{0 t}} & \cdots & \frac{\lambda}{q_{0 t}} \\
\frac{\lambda}{q_{0 t}} & -\frac{\lambda}{q_{1 t}} & 0 & \cdots & 0 \\
\frac{\lambda}{q_{0 t}} & 0 & -\frac{\lambda}{q_{2 t}} & \cdots & 0 \\
\cdots & \ldots & \cdots & \cdots & \ldots \\
\frac{\lambda}{q_{0 t}} & 0 & 0 & \cdots & -\frac{\lambda}{q_{m t}}
\end{array}\right) .
\end{aligned}
$$

Next, we show that the Hessian matrix $H\left(\psi\left(x_{t}, p_{t}\right)\right)$ is negative semidefinite. Let $Z=\left(z_{0}, z_{1}, \ldots, z_{m}\right) \neq 0$; then

$$
\begin{aligned}
& Z^{T} H\left(\psi\left(x_{t}, p_{t}\right)\right) Z \\
& \quad=-\lambda\left[z_{0}^{2} \sum_{j=1}^{m} \frac{q_{j t}}{\left(q_{0 t}\right)^{2}}+\sum_{j=1}^{m} \frac{z_{j}^{2}}{q_{j t}}-\frac{2}{q_{0 t}} z_{0} \sum_{j=1}^{m} z_{j}\right] .
\end{aligned}
$$

If $z_{0} \sum_{j=1}^{m} z_{j} \leq 0$, then $Z^{T} H\left(\psi\left(x_{t}, p_{t}\right)\right) Z \leq 0$. This implies that $H\left(\psi\left(x_{t}, p_{t}\right)\right)$ is negative semidefinite. Therefore, we let $z_{0} \sum_{j=1}^{m} z_{j}>0$. If $z_{0}>0$ and $\sum_{j=1}^{m} z_{j}>0$, then

$$
\begin{aligned}
& -Z^{T} H\left(\psi\left(x_{t}, p_{t}\right)\right) Z \\
& \quad \geq \frac{2 \lambda z_{0}}{q_{0 t}}\left[\sqrt{\left(\sum_{j=1}^{m} q_{j t}\right)\left(\sum_{j=1}^{m} \frac{z_{j}^{2}}{q_{j t}}\right)}-\sum_{j=1}^{m} z_{j}\right] .
\end{aligned}
$$

Since

$$
\begin{aligned}
& \sqrt{\left(\sum_{j=1}^{m} q_{j t}\right)\left(\sum_{j=1}^{m} \frac{z_{j}^{2}}{q_{j t}}\right)} \\
& =\sqrt{\sum_{j=1}^{m} z_{j}^{2}+2 \sum_{i \neq j}\left(\frac{q_{i t}}{q_{j t}} z_{j}^{2}+\frac{q_{j t}}{q_{i t}} z_{i}^{2}\right)} \\
& \geq \sqrt{\sum_{j=1}^{m} z_{j}^{2}+2 \sum_{i \neq j} z_{i} z_{j}}=\sum_{j=1}^{m} z_{j},
\end{aligned}
$$

it follows that $-Z^{T} H\left(\psi\left(x_{t}, p_{t}\right)\right) Z \geq 0$. This implies that $H\left(\psi\left(x_{t}, p_{t}\right)\right)$ is negative semidefinite.

Similarly, we can conclude that $H\left(\psi\left(x_{t}, p_{t}\right)\right)$ is negative semidefinite, if $z_{0}<0$ and $\sum_{j=1}^{m} z_{j}<0$. It directly follows that $\psi\left(x_{t}, p_{t}\right)$ is concave in $q_{t}$.

Here we show that $\psi\left(x_{t}, p_{t}\right)$ is concave in $q_{t}$ so that we can investigate the property of optimal pricing strategy in the easier way. We proceed to analyze the property of the optimal prices.

Theorem 5. For a given remaining seat level $x_{t}$ in period $t$, the optimal price of flight $i$ is set to

$$
p_{i t}^{*}\left(x_{t}\right)=\Delta_{x_{i}} V_{t-1}\left(x_{t}\right)+\eta\left(t, x_{t}\right),
$$

where $\eta\left(t, x_{t}\right)$ is the unique solution to $\left[\eta\left(t, x_{t}\right)-1\right] e^{\eta\left(t, x_{t}\right)}-$ $\sum_{j=1}^{m} e^{a_{j}+\Lambda_{j}-\Delta_{x_{j}} V_{t-1}\left(x_{t}\right)}=0$.

Furthermore, the optimal expected revenue is given by

$$
V_{t}^{*}\left(x_{t}\right)=\lambda \sum_{h=1}^{t}\left[\eta\left(h, x_{t}\right)-1\right]
$$

Proof. From Theorem $4, \psi\left(x_{t}, p_{t}\right)$ is concave in $q_{t}$. In order to obtain the optimal price $p_{j t}$, we use the first-order condition $\partial \psi\left(x_{t}, p_{t}\right) / \partial q_{j t}=0$; that is,

$$
\begin{aligned}
& \frac{\partial \psi\left(x_{t}, p_{t}\right)}{\partial q_{j t}} \\
& \quad=\lambda\left[a_{j}+\Lambda_{j}-\ln \left(q_{j t}\right)+\ln \left(1-\sum_{k=1}^{m} q_{k t}\right)\right. \\
& \left.\quad-\Delta_{x_{j}} V_{t-1}\left(x_{t}\right)-\frac{1}{1-\sum_{k=1}^{m} q_{k t}}\right]=0 .
\end{aligned}
$$

Substituting $\eta\left(t, x_{t}\right)=1 /\left(1-\sum_{k=1}^{m} q_{k t}\right)$ into (38), we can get

$$
q_{j t}=\frac{1}{\eta\left(t, x_{t}\right)} e^{a_{j}+\Lambda_{j}-\eta\left(t, x_{t}\right)-\Delta_{x_{j}} V_{t-1}\left(x_{t}\right)} .
$$

Therefore, $\sum_{j=1}^{m} q_{j t}=\left(1 / \eta\left(t, x_{t}\right)\right) \sum_{j=1}^{m} e^{a_{j}+\Lambda_{j}-\eta\left(t, x_{t}\right)-\Delta_{x_{j}} V_{t-1}\left(x_{t}\right)}$. It follows that

$$
\left[\eta\left(t, x_{t}\right)-1\right] e^{\eta\left(t, x_{t}\right)}-\sum_{j=1}^{m} e^{a_{j}+\Lambda_{j}-\Delta_{x_{j}} V_{t-1}\left(x_{t}\right)}=0,
$$

due to $\sum_{j=1}^{m} q_{j t}=1-1 / \eta\left(t, x_{t}\right)$. Next, we show that $\eta\left(t, x_{t}\right)$ is the unique solution to $(40)$.

Let $\Omega(\xi)=(\xi-1) e^{\xi}-\sum_{j=1}^{m} e^{a_{j}+\Lambda_{j}-\Delta_{x_{j}} V_{t-1}\left(x_{t}\right)}$; then we can conclude that $\Omega(1)<0$ and $\Omega(+\infty)=+\infty$. Since $\partial \Omega(\xi) / \partial \xi=\xi e^{\xi}>0$ for any $\xi \in(1,+\infty)$, it follows that $\Omega(\xi)$ is monotonically increasing in $\xi$ for $\xi \in(1,+\infty)$. This indicates that $\eta\left(t, x_{t}\right) \in(1,+\infty)$ is the unique solution to $(40)$; that is, $\sum_{k=1}^{m} q_{k t}<1$. From (25) and (39), we can derive

$$
p_{i t}^{*}=\eta\left(t, x_{t}\right)+\Delta_{x_{i}} V_{t-1}\left(x_{t}\right)
$$

where $\eta\left(t, x_{t}\right)$ is the unique solution to $\left[\eta\left(t, x_{t}\right)-1\right] e^{\eta\left(t, x_{t}\right)}-$ $\sum_{j=1}^{m} e^{a_{j}+\Lambda_{j}-\Delta_{x_{j}} V_{t-1}\left(x_{t}\right)}=0$.

Substituting (41) into (28), we have

$$
V_{t}^{*}\left(x_{t}\right)=\lambda\left[\eta\left(t, x_{t}\right)-1\right]+V_{t-1}\left(x_{t}\right)
$$

Applying the boundary conditions, we can get

$$
V_{t}^{*}\left(x_{t}\right)=\lambda \sum_{h=1}^{t}\left[\eta\left(h, x_{t}\right)-1\right] \text {. }
$$




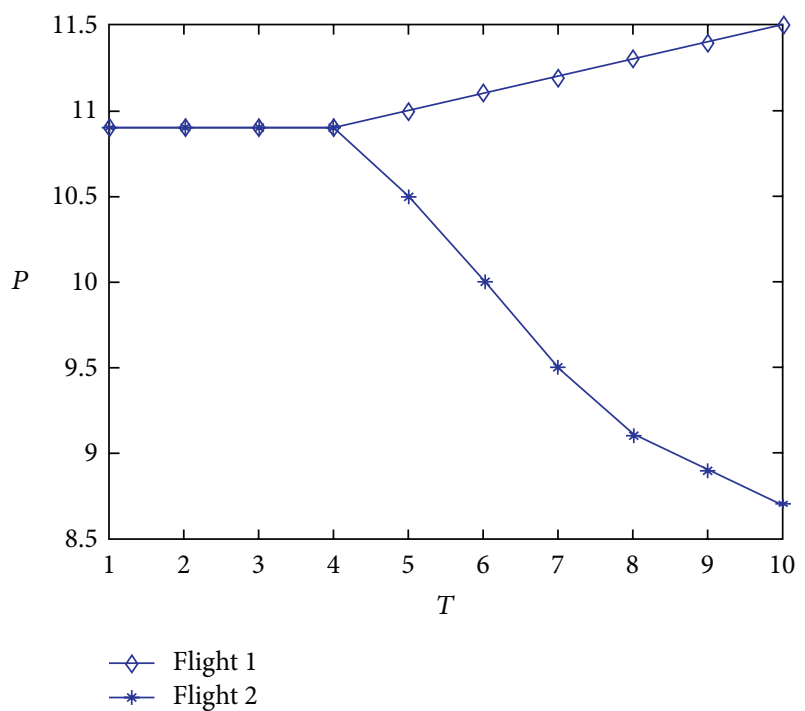

FIGURE 1: Optimal prices of both flights in time remaining $t$.

\section{Numerical Examples}

In this section, we numerically illustrate the effect of passenger mental accounting on the optimal pricing of flights. We examine sets of examples with two substitutable flights in which $a_{1}=10, a_{2}=6, \lambda=0.7$, initial seat levels $c_{1}=4, c_{2}=6$, and corresponding mental accounting depths $\gamma_{1}=8$ and $\gamma_{2}=6$. The mental accounting coefficients that a passenger derives from choosing flights 1 and 2 are $b_{1}=0.4$ and $b_{2}=0.6$, respectively.

Figure 1 depicts the optimal prices for both flights with respect to remaining time, at fixed seat levels $c_{1}=4, c_{2}=6$. In Figure 1, we observe time monotonicity, the optimal price of flight 1 increases in remaining time $t$, and the optimal price of flight 2 decreases in remaining time $t$. Moreover, the optimal prices of both flights converge to the identical value. This means that the uniform pricing strategy begins to take effect when the remaining seat level is abundant relative to passenger demand.

Figure 2 illustrates the optimal prices of both flights as a function of the remaining seat level of flight 1 in period 10, with the remaining seat level of flight 2 fixed as $c_{2}=6$. The optimal price of flight 1 is nonincreasing with respect to its own remaining seat level, and the optimal price of flight 2 is nonmonotonic in the remaining seat level of flight 1.

Next, we examine how the mental accounting depth affects the optimal prices of both flights. Specifically, we want to depict the change in the optimal prices of both flights over mental accounting depth when $t=10, c_{1}=4$, and $c_{2}=6$. In this numerical simulation, we keep the depth of mental accounting 2 constant; that is, $\gamma_{2}=6$. The depth of mental accounting 1 can be chosen from 1 to 10 . Figure 3 gives the optimal prices of both flights for each depth level of mental accounting 1. From Figure 3, we observe that mental accounting depth significantly affects the optimal prices of both flights. Given the remaining seat level and remaining

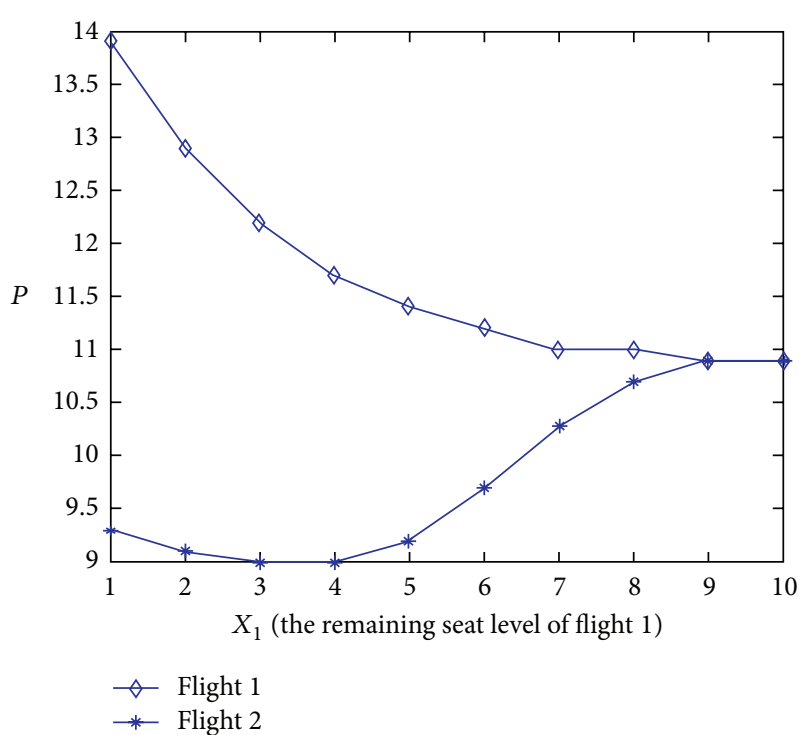

FIGURE 2: Optimal prices of both flights in the remaining seat level of flight 1 .

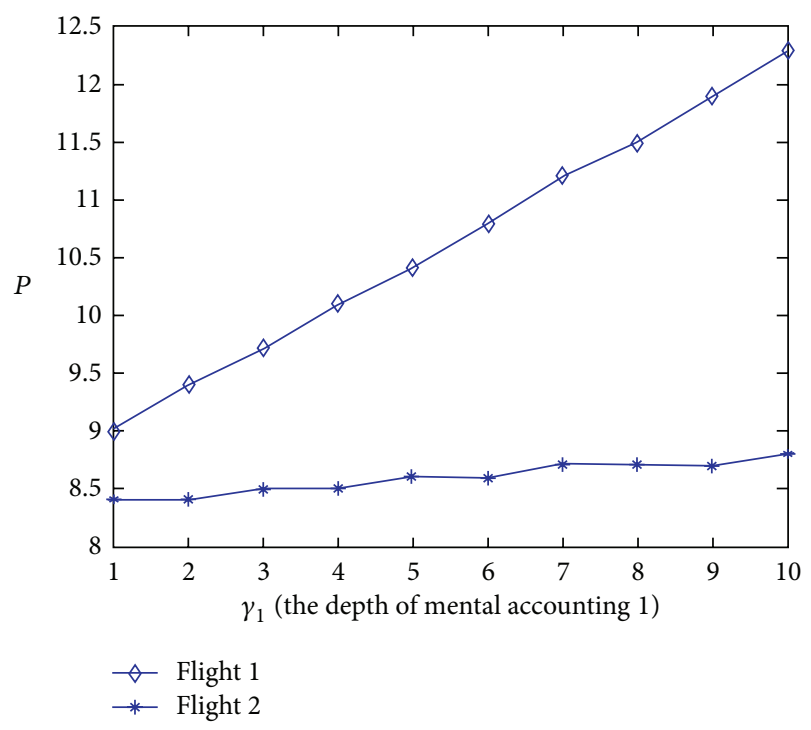

FIGURE 3: Effect of mental accounting on the optimal prices.

time, the optimal prices of both flights are nondecreasing with the depth level of mental accounting 1.

\section{Conclusion}

In this paper, we study the dynamic pricing problems for a single flight and multiple flights over finite booking horizon in the presence of mental accounting. We use Bellman equation to obtain the optimal prices. Furthermore, we present the numerical simulation to investigate how mental accounting depth affects the optimal prices. According to the numerical example, we derive that passenger's mental accounting has a positive impact on the optimal prices. 
In the future study, we can incorporate the batch demand into dynamic pricing instead of unit purchase. Of course, it is also useful to incorporate competition into the dynamic pricing problem or consider demand learning in our model.

\section{Conflict of Interests}

The authors declare that there is no conflict of interests about the named companies regarding the publication of this paper.

\section{Acknowledgments}

This work was supported by the National Natural Science Foundation of China (nos. 71172172 and 71272058), Specialized Research Fund for Doctoral Program of Higher Education of China (no. 20121101110054), Special Program for International Science, and Technology Cooperation of Beijing Institute of Technology (no. GZ2014215101).

\section{References}

[1] K. Talluri and G. van Ryzin, "Revenue management under a general discrete choice model of consumer behavior," Management Science, vol. 50, no. 1, pp. 15-33, 2004.

[2] K. Talluri and G. van Ryzin, The Theory and Practice of Revenue Management, Kluwer Academic, Boston, Mass, USA, 2004.

[3] D. Zhang and W. L. Cooper, "Revenue management for parallel flights with customer-choice behavior," Operations Research, vol. 53, no. 3, pp. 415-431, 2005.

[4] W. Elmaghraby and P. Keskinocak, "Dynamic pricing in the presence of inventory considerations: research overview, current practices, and future directions," Management Science, vol. 49, no. 10, pp. 1287-1309, 2003.

[5] Y. Feng and B. Xiao, "A dynamic airline seat inventory control model and its optimal policy," Operations Research, vol. 49, no. 6, pp. 938-949, 2001.

[6] S. Chen, G. Gallego, M. Z. F. Li, and B. Lin, "Optimal seat allocation for two-flight problems with a flexible demand segment," European Journal of Operational Research, vol. 201, no. 3, pp. 897-908, 2010.

[7] D. Besanko and W. L. Winston, "Optimal price skimming by a monopolist facing rational consumers," Management Science, vol. 36 , no. 5 , pp. 555-567, 1990.

[8] R. H. Thaler, "Mental accounting and consumer choice," Marketing Science, vol. 27, no. 1, pp. 15-25, 2008.

[9] Z.-J. M. Shen and X. Su, "Customer behavior modeling in revenue management and auctions: a review and new research opportunities," Production and Operations Management, vol. 16, no. 6, pp. 713-728, 2007.

[10] X. Su, "Bounded rationality in newsvendor models," Manufacturing and Service Operations Management, vol. 10, no. 4, pp. 566-589, 2008.

[11] I. Popescu and Y. Wu, "Dynamic pricing strategies with reference effects," Operations Research, vol. 55, no. 3, pp. 413-429, 2007.

[12] J. Nasiry and I. Popescu, "Dynamic pricing with loss-averse consumers and peak-end anchoring," Operations Research, vol. 59, no. 6, pp. 1361-1368, 2011.
[13] D. Zhang and W. L. Cooper, "Pricing substitutable flights in airline revenue management," European Journal of Operational Research, vol. 197, no. 3, pp. 848-861, 2009.

[14] Y. Akçay, H. P. Natarajan, and S. H. Xu, "Joint dynamic pricing of multiple perishable products under consumer choice," Management Science, vol. 56, no. 8, pp. 1345-1361, 2010.

[15] K. Y. Lin and S. Y. Sibdari, "Dynamic price competition with discrete customer choices," European Journal of Operational Research, vol. 197, no. 3, pp. 969-980, 2009.

[16] M. Suh and G. Aydin, "Dynamic pricing of substitutable products with limited inventories under logit demand," IIE Transactions (Institute of Industrial Engineers), vol. 43, no. 5, pp. 323-331, 2011.

[17] L. Dong, P. Kouvelis, and Z. Tian, "Dynamic pricing and inventory control of substitute products," Manufacturing and Service Operations Management, vol. 11, no. 2, pp. 317-339, 2009.

[18] Y. Aviv and A. Pazgal, "Optimal pricing of seasonal products in the presence of forward-looking consumers," Manufacturing and Service Operations Management, vol. 10, no. 3, pp. 339-359, 2008.

[19] Y. Levin, J. McGill, and M. Nediak, "Dynamic pricing in the presence of strategic consumers and oligopolistic competition," Management Science, vol. 55, no. 1, pp. 32-46, 2009.

[20] Y. Levin, J. McGiil, and M. Nediak, "Optimal dynamic pricing of perishable items by a monopolist facing strategic consumers," Production and Operations Management, vol. 19, no. 1, pp. 4060, 2010.

[21] T. Levina, Y. Levin, J. McGill, and M. Nediak, "Dynamic pricing with online learning and strategic consumers: an application of the aggregating algorithm," Operations Research, vol. 57, no. 2, pp. 327-341, 2009.

[22] Y. Chen and Z. J. Zhang, "Dynamic targeted pricing with strategic consumers," International Journal of Industrial Organization, vol. 27, no. 1, pp. 43-50, 2009.

[23] S. Liao and H. Chu, "Influence of consumer online resale awareness on purchase decisions: a mental accounting perspective," European Journal of Marketing, vol. 47, no. 10, pp. 1576-1597, 2013.

[24] S. Erat and S. R. Bhaskaran, "Consumer mental accounts and implications to selling base products and add-ons," Marketing Science, vol. 31, no. 5, pp. 801-818, 2012.

[25] L. Chen, A. G. Kök, and J. D. Tong, "The effect of payment schemes on inventory decisions: the role of mental accounting," Management Science, vol. 59, no. 2, pp. 436-451, 2013.

[26] S. P. Anderson, A. de Palma, and J.-F. Thisse, Discrete Choice Theory of Product Differentiation, MIT Press, Cambridge, Mass, USA, 1992. 


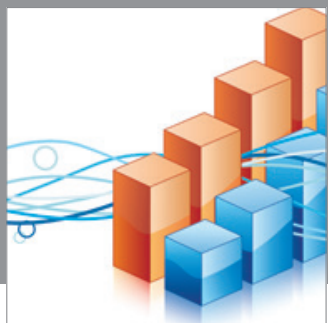

Advances in

Operations Research

mansans

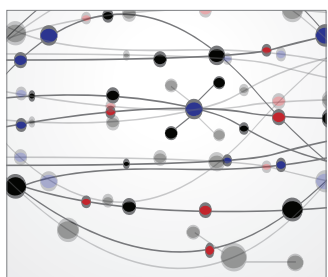

The Scientific World Journal
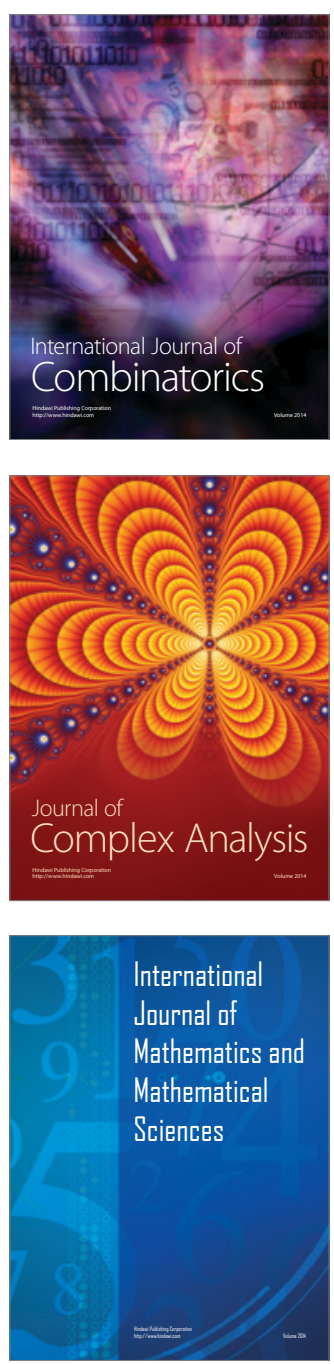
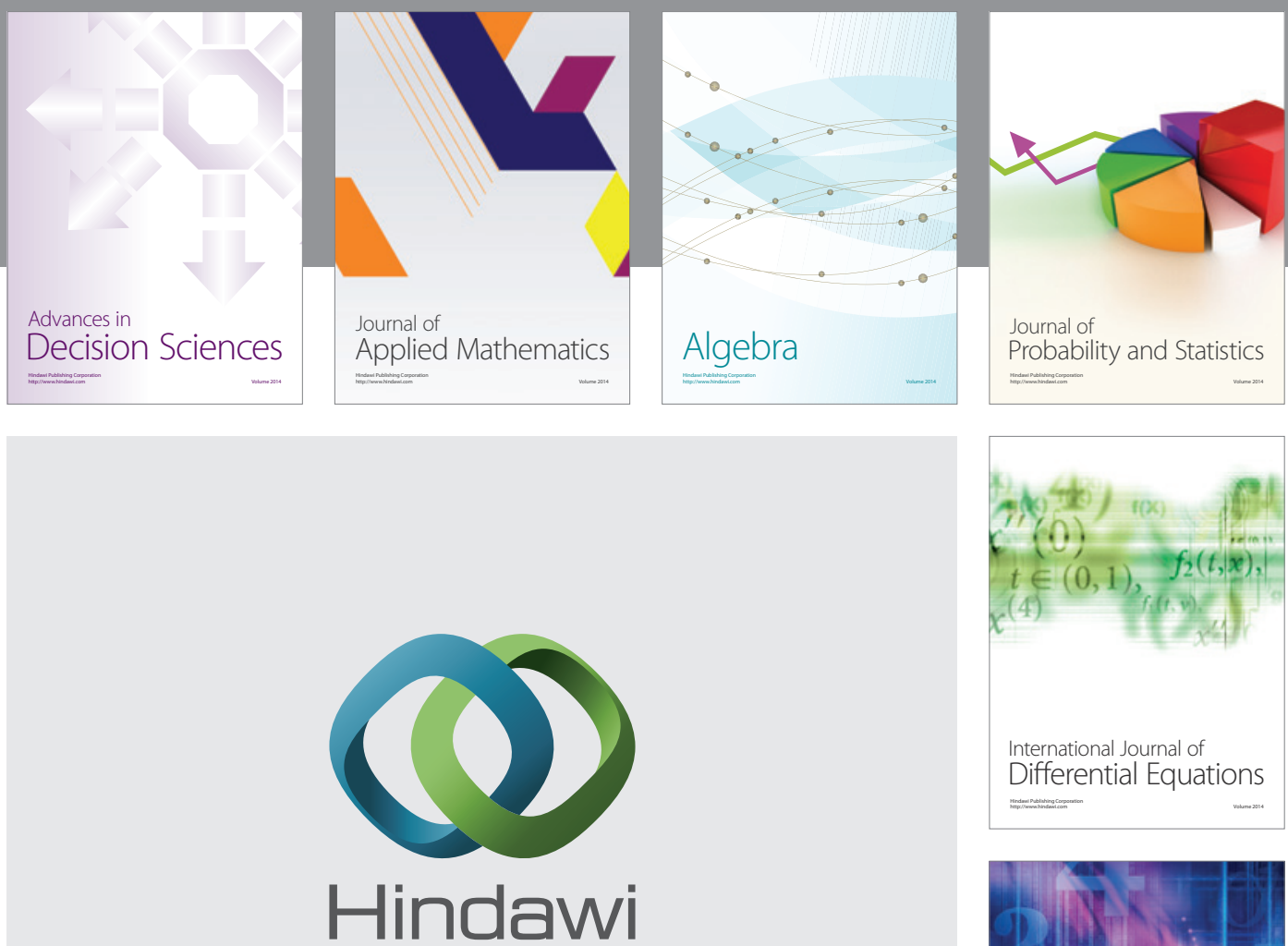

Submit your manuscripts at http://www.hindawi.com
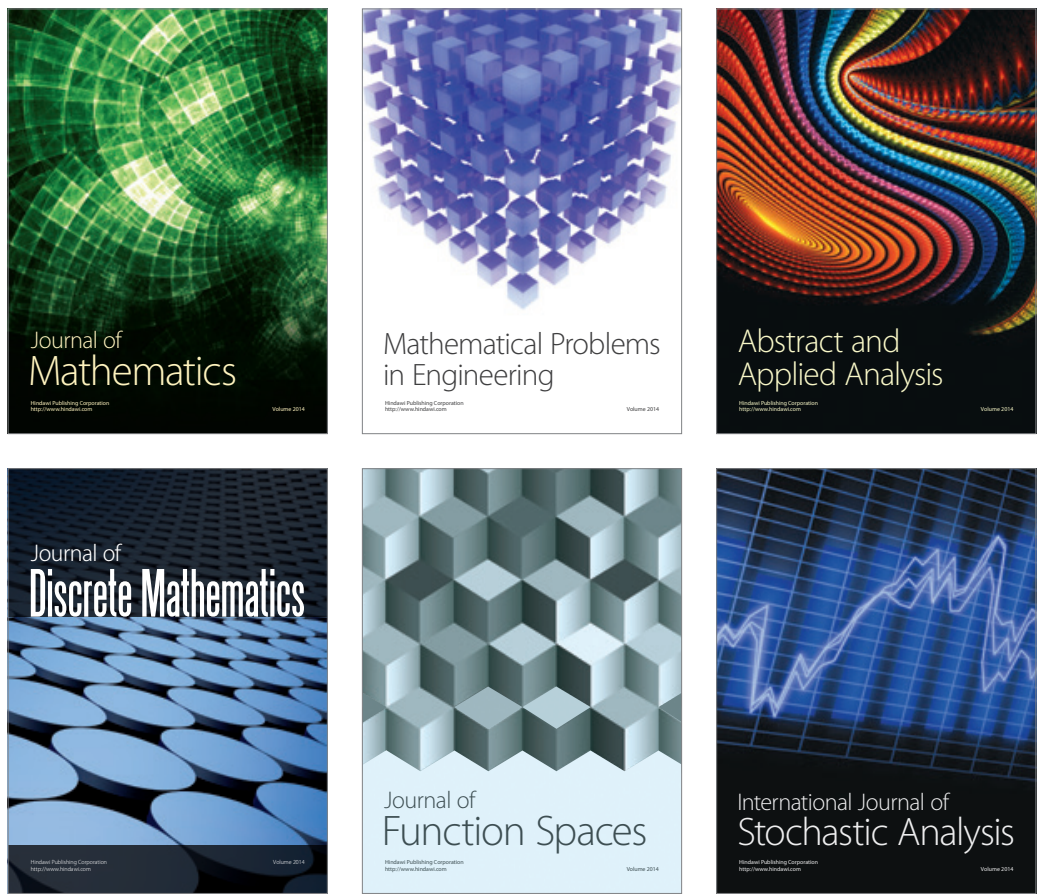

Journal of

Function Spaces

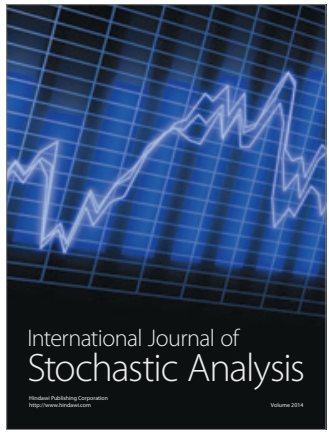

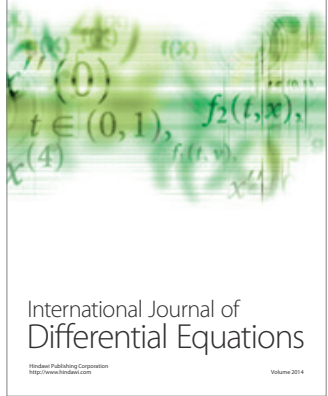
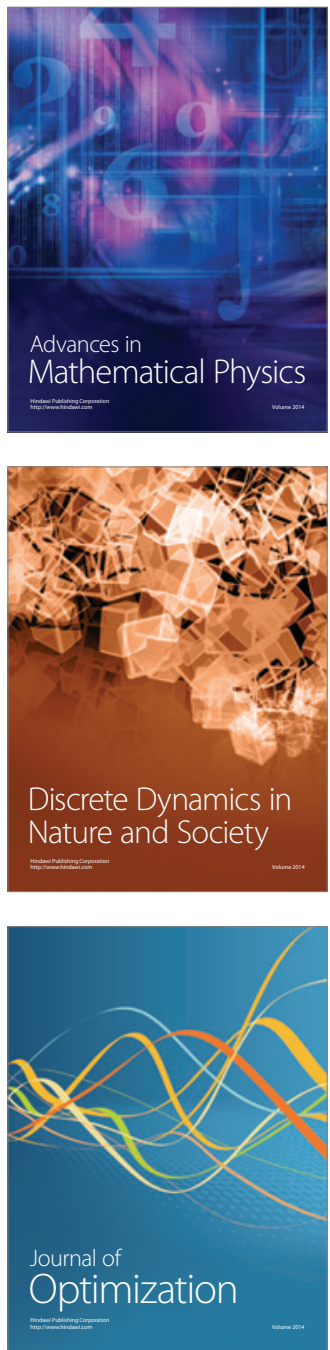